\title{
Narrated animated solution videos in a mastery setting
}

\author{
Noah Schroeder," Gary Gladding, Brianne Gutmann, and Timothy Stelzer \\ Department of Physics, University of Illinois Urbana Champaign, Urbana, Illinois 61801, USA
}

(Received 28 August 2014; published 2 February 2015)

\begin{abstract}
Narrated animated solution videos were implemented in a clinical study that compared a mastery setting that employed repeated cycles of testing with instructional support to a group that had a single opportunity to experience the materials. The mastery setting students attempted sequential questions sets on a topic, with animated solutions between each set, until mastery was achieved, combining formative assessment with worked examples. Students showed significant improvement from their first to second tries on similar sets of problems, attributable to the feedback and solutions they were given after the first try. These improvements were shown in two topics, superposition and electric potential. The single try group was given one version of the questions and solutions, and while they were not required to watch the solutions to move forward, they chose to. On a post-test including near and far transfer questions, no significant difference was seen between the mastery group and the single try group, but both significantly outperformed a control group that received no instructional support, indicating that students successfully transferred the skills from the solutions to the post-test.
\end{abstract}

DOI: 10.1103/PhysRevSTPER.11.010103

PACS numbers: 01.40.Fk

\section{INTRODUCTION}

Worked examples in the form of solutions to problems have a long history in education research [1]. Contrary to classic instruction by problem solving, worked example based instruction extends the learning period where students take in information about successful strategies from fully completed solutions. Cognitive load theory states that when novice students attempt problem solving they often employ inefficient strategies, such as means-end analysis, that use up working memory, which hampers the acquisition of problem solving schemas [2]. Worked examples help students "attend to problem states and their associated moves rather than searching for the right moves involved in conventional problem solving" [3]. Students can spend their resources on activities like studying procedures and learning domain principles. The improved learning from this type of instructions has been called "the worked example effect" [4]. Teaching strategies that stress the use of worked examples have been shown to be more effective than problem solving in areas like middle school mathematics [5], algebra [6], and physics [7]. Novice students prefer this method of instruction as well $[8,9]$. Novices in a topic learn expertlike strategies from exposure to completed solutions to problems. However, when experts use worked examples, the advantages are limited, and can sometimes even go so far as to be detrimental to the

\section{*schroe16@illinois.edu}

Published by the American Physical Society under the terms of the Creative Commons Attribution 3.0 License. Further distribution of this work must maintain attribution to the author(s) and the published article's title, journal citation, and DOI. learning process. This is called the expertise reversal effect [10]. Experts in a topic have the ability to group sets of steps in a pattern into chunks [11]. Redundancy in the steps to a solution can increase cognitive load for experts because reading each step can distract them from forming patterns. Worked examples are best when fine tuned to the learner's skill level.

The design of worked examples has been investigated in the past $[12,13]$. Much of the research done on worked examples has been done on static solutions, that is, prepared detailed work that is written or printed out on a piece of paper. Multimedia design for education has been investigated more so in recent times as technology encourages uses of animations [14,15]. Mayer and Moreno give many ways to reduce cognitive load in a multimedia environment, such as moving information from the visual to the auditory channel and avoiding redundancy between printed and spoken words [16]. Multimedia learning modules have been shown to be more effective than textbooks in introducing new materials to students, regardless of student background or ability $[17,18]$.

Along with worked examples this experiment will make heavy use of formative assessment for students. Formative assessment has been shown to have vast, powerful effects on student learning [19]. Frequent feedback on low stakes assessments helps inform students how they are doing and what they should do to improve. Frequent testing has been shown to improve recall of facts [20]. This recall is maximized when students are given delayed feedback, that is, they are told which questions they got correct or incorrect after they finish a set of tasks [21]. Mastery learning, whereby students are required to attain a certain level of success in a topic before moving on, provides a 
fertile model for combining formative assessment and worked examples. Mastery learning has been shown to have positive effects on student learning over many different studies in many different topics [22].

This paper describes results from a clinical experiment designed to study the feasibility of implementing scalable web-based mastery activities for introductory physics. Specifically, the study provides data on three components essential for a successful implementation. First, does the mastery format with animated solutions provide sufficient support for students to succeed in completing the activity with the specified proficiency? Second, does the mastery format change student behavior compared with students that are not required to achieve a specified proficiency to complete the assignment. Third, are students able to apply the knowledge learned in mastery to new problems in which it is applicable?

\section{PROCEDURE}

Students in our experiment were separated into three treatment groups, the "mastery" group that attempted the learning materials in a mastery setting, the "single try" group that only attempted 1 version of each topic before moving on, and a control group, that was given no learning intervention. The mastery group sat at a computer, with pencil and paper, and attempted a set of questions which were delivered online. Students received short instruction as to how the materials would be presented before starting the learning process. Students attempted the questions in a closed book environment, accompanied only by the equation sheet they would be given on exams. Students answered all of the questions in a set before feedback was given. The student answers were recorded in an online database. After submitting the set, students were given feedback on which questions they answered correctly, and were given an opportunity to view a narrated animated solution video to each question online. The solutions contained step-by-step instructions particular to each question. Students were free to choose which animated solutions they would watch and which they would skip, as well as how much time they would spend reviewing each solution. By structuring our experiment this way, we hoped to avoid the expertise reversal effect. Students that did well knew they did well, and could avoid redundant explanations of material they already understood. Students then indicated they were finished viewing the solutions. If they had not achieved mastery, another set of questions was presented on the same topic. Each new question in the second set was closely related to a question from the first set. To ensure students were completing the second set using information they had learned, and not reading off equations from the first set of animated solutions, solutions to the previous set of questions were not accessible during the testing period for the second set, and they were told not to use notes during this period. After completing the second set, students submitted their answers for grading, and solutions to the second set were made available to the students, just as had been done on the first set. Students continued in this question-solution pattern until mastering the question set, here defined as having $85 \%$ of the questions correct. Students were informed of this expectation before starting the experiment. If a student mastered the first set of questions they saw on the first topic, that student was presented with solutions and given an opportunity to view them, and when finished, moved on to the second topic. A repeated cycle of testing and presentation of solutions was used to maximize the formative assessment given along with presenting relevant multimedia solutions at the right time, to render the most efficient use of students' time. After mastering the second topic, the student was finished with the learning phase of this experiment. They then moved on to a post-test that consisted of 10 questions pertaining to potential difference in planar and spherical geometries. Each student completed the experiment in one sitting.

The second group, the single try group, was given a modified, simpler version of the primary treatment. The second group was only given the first set of questions for the first topic. They attempted the questions, had their answers evaluated, and were told which ones they answered correctly and which ones incorrectly. They were then given access to the solutions to that first set, and then, no matter their performance on that first set, moved on to the second topic, where they attempted the first set and then were shown the solution videos to that set. At that point the students were finished with the learning phase of the experiment and moved on to the post-test. The final group, the control group, attempted the post-test without any intervention. Results from this group served as "pretest," from which we could measure the effective learning gains by the other two groups, without asking them to perform identical problems before and after the treatment.

The post-test was graded by four graders, with each question graded on a scale of 0 to 3 . Post-tests were graded without knowledge of which treatment the student received. The grades given by the four graders were compared, and any scores that differed by more than 1 point were discussed to resolve the discrepancy. The average of the 4 scores was assigned as the final grade for each student on each question. That score will be used in the results.

\section{MATERIALS}

Two topics were used in this experiment: superposition of electric fields, and electric potential difference. All question sets were given in a multiple choice format. Students were first shown a question set with eight questions on superposition of electric fields with planar charge sheets and conducting slabs. The superposition material included finding the electric field due to two and three charge sources and also finding the surface charge 
density on a conducting slab. After mastering this material, students moved on to a set of nine questions on electric potential difference. The potential difference material included finding the potential difference between two points when given the functional form of the electric field between the points, including piecewise definitions, as well as a set of six spherical geometry questions that asked students to find the electric field at certain points and the potential difference between certain points given a charge distribution. For both topics, four sets of questions were created, as well as solutions for each question. In total, sixty-eight narrated animated solutions were created for this experiment. The question sets are attached in the Supplemental Material [23]. Student performance on the first and second sets of each topic will be shown below. The solutions were created and animated using Powerpoint graphics and animation, and were narrated and recorded using Jing. Solutions were designed with multimedia principles in mind [24]. Several screenshots of the final frame of some representative solutions have been included in the Supplemental Material [23].

The post-test consisted of ten questions that all pertained to potential difference. The post-test is included in the Supplemental Material [23]. The first four questions required students to calculate a potential difference in a planar geometry. The fifth required students to work backwards from a given potential difference to find a surface charge density on an infinite sheet. The sixth and seventh questions asked students to compare potential differences given different positions and thicknesses of a charged conductor. The eighth question was a potential difference calculation in spherical geometry. The ninth and tenth questions asked students to compare potential differences for various spherical geometries. The post-test was given on paper and pencil, not online.

The materials we used were relevant to students in two different phases of their learning cycle within their actual physics course. This experiment was performed on a weekend, 10 days before the first midterm exam. The material on superposition had already been lectured upon and also covered in homework, lab, and the discussion section. The potential difference materials had been lectured upon, but homework, lab, and discussion sections on the topic had not yet occurred. Students had not yet had much exposure to working problems in that area. As will be seen, student performance on the superposition material was higher in their first attempt than on their first attempt for the potential difference, and part of this is due to the scheduling of our experiment.

\section{POPULATION}

The experiment was done in the calculus-based course University Physics: Electricity \& Magnetism at the University of Illinois at Urbana-Champaign. This course is the second physics course required for physics and engineering majors and is usually taken during the second year at university. All students in the course were invited by email to participate in the experiment. Volunteers signed up by an online form. No credit was offered to the students for this experiment. The students were told that if they completed the experiment, they would have access to an extra office hour provided by experienced TAs. Eightyeight students signed up. These students were randomly split into three groups as discussed earlier: the mastery group that was required to master the material $(85 \%)$ via successive sets on each topic with solutions presented after each set, the single try group that attempted one set of questions on each topic with solutions after finishing, and a control group.

\section{RESULTS}

We now present results on student behavior during the learning phase for the mastery group. Figure 1 shows the performance of these students on each question in their first attempt at the superposition materials, the first materials they saw in the experiment.

Students attempted these questions before seeing any of the learning materials. These scores on the first set of questions show what students knew about superposition of electric fields from planar charge sources as they began our experiment. The average question score was $67 \%$. Figure 2 shows the distribution of set scores among the 24 students.

Students had to get at least 7 of the 8 questions correct in order to move on, and nine students did so on their first attempt. The remaining 15 were given access to the solutions, and moved on to attempt the second set of questions on superposition. As shown in Fig. 2, on the second set, 11 of the remaining 15 students mastered the material, leaving only 4 students requiring a third set. All four remaining students mastered the superposition material on their third try. The high rate of achievement on the 2nd and 3rd attempts suggests the narrated solutions provided sufficient support for students to achieve mastery

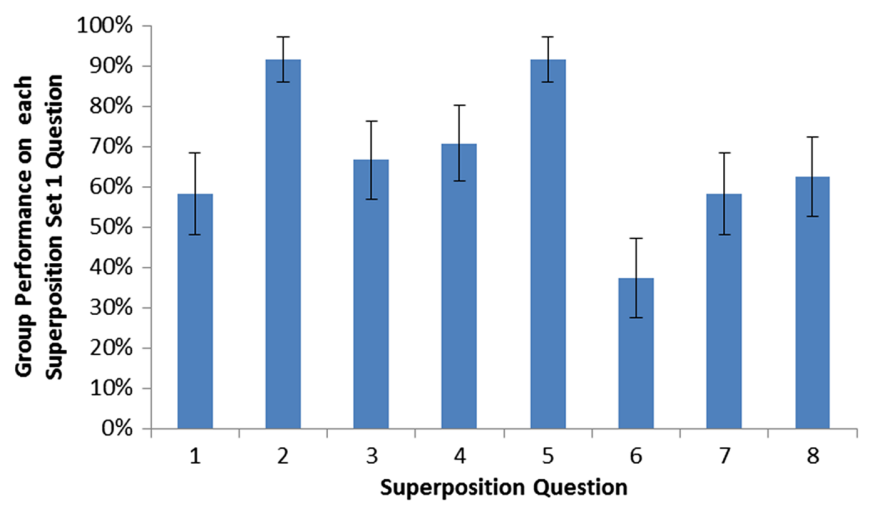

FIG. 1. Percentage of 24 students that got each question correct on the first superposition set. 


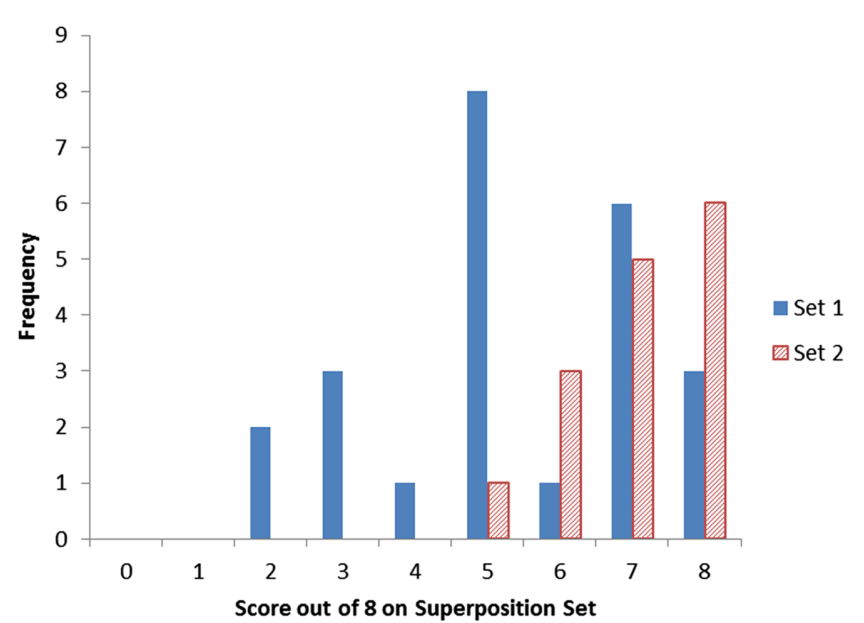

FIG. 2. Distribution of scores, out of 8, superposition sets. Our criteria for mastering the material was $85 \%$, which meant that a student needed to get a score of 7 or 8 to master the material. Nine out of the 24 students achieved that goal on the first set and moved on to the next topic after being given a chance to view the animated solutions, and therefore are not included in the scores for the second set.

without requiring external interventions. This can also be seen on a question by question basis.

Figure 3 shows the performance of the 15 remaining students on set 2 compared to their performance on set 1 . The total scores are shown in Table I. We found improvement on 7 of the 8 questions. When we compare the total score on sets 1 and 2 for superposition for the students who had to do a second set, we find a significant improvement from $53 \%$ to $88 \%$.

After mastering the superposition material, students moved on to the problem sets on potential difference. One student had time constraints and had to leave before finishing, and is not included in this analysis. The performance of the 23 remaining students on the first set of questions on potential difference is shown in Fig. 4.

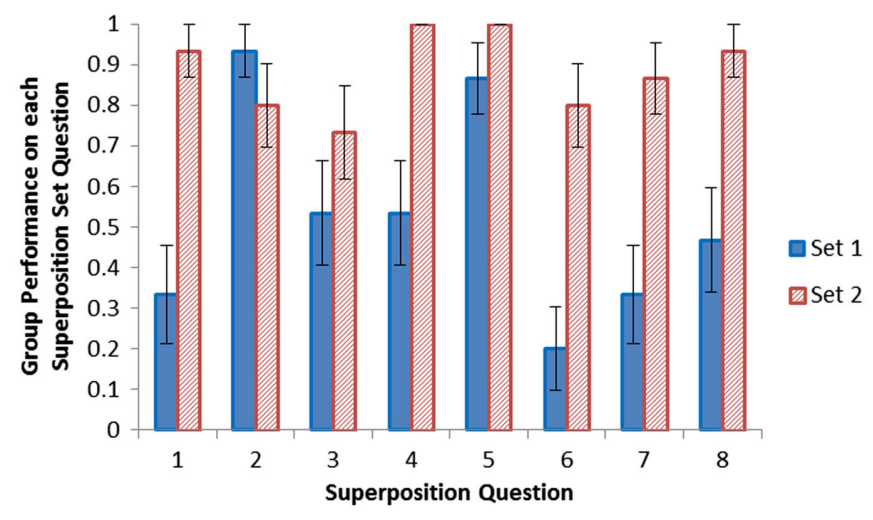

FIG. 3. Scores on questions for sets 1 and 2 of the topic superposition. This only includes the 15 students who did not master set 1. Learning gains were shown for 5 questions.
TABLE I. Averages on sets 1 and 2 for both topics, including only the students that did not master set 1 of a given topic.

\begin{tabular}{lccccc}
\hline \hline \multicolumn{5}{c}{ Results for those who did not master on first try } \\
\hline Set 1 & Set 2 & tStat & df & P \\
\hline Superposition & $53 \% \pm 4.1 \%$ & $88 \% \pm 3.1 \%$ & 7.15 & 14 & $<0.001$ \\
Potential difference & $37 \% \pm 3.0 \%$ & $78 \% \pm 4.8 \%$ & 8.48 & 19 & $<0.001$ \\
\hline \hline
\end{tabular}

The average score on this set of questions was $44 \%$. Questions 1-3 involved finding the potential difference between two points when given the functional form of the electric field. Students performed poorly, averaging only $17 \%$ on this section. Students came in with little knowledge of how to go from electric field to potential difference. Questions 4-6 involved finding the electric field in spherical geometry. Students performed very well on this material as compared to the prior 3 questions, averaging $83 \%$ on their first try. Questions 7-9 asked for potential differences in the same spherical geometry as questions 4-6. This required incorporating knowledge of the form of the electric field in that region with calculating potential differences from electric fields. Students performed poorly at this task, which comes as no surprise since they performed so poorly on questions 1-3. Question 7's higher score of $48 \%$ could be due to students resorting to the familiar $\mathrm{kq} / \mathrm{r}$ equation. This equation failed them for questions 8 and 9. The timing of the experiment may explain the discrepancy between the scores on questions relevant to potential difference versus those relevant to electric fields. The experiment took place after the homework on electric fields was due and their discussions on the topic had been completed, but before the potential difference homework was due. Students appeared to come to the experiment fluent in calculating electric fields in a spherical geometry, but were deficient in potential difference calculations.

A histogram of the number of questions each student got correct for sets 1 and 2 is shown in Fig. 5. Three of the 23

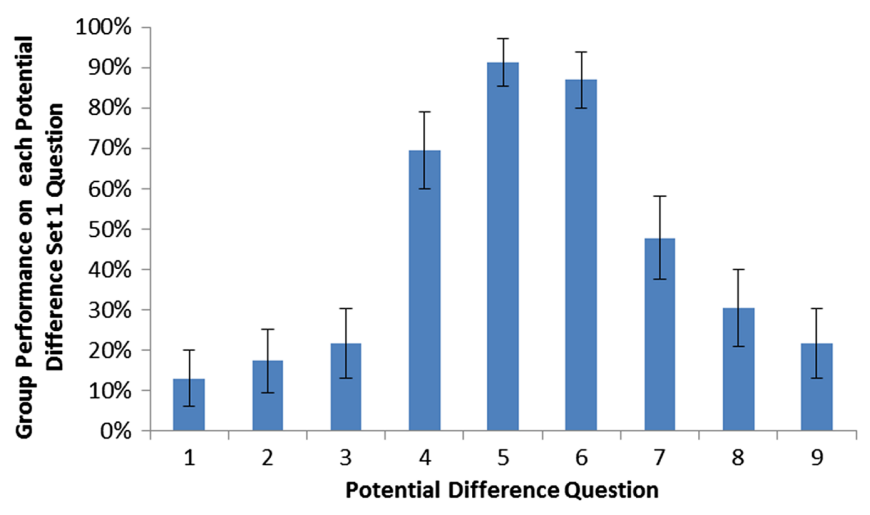

FIG. 4. Percentage of 23 students that got each question correct on the first potential difference set. 


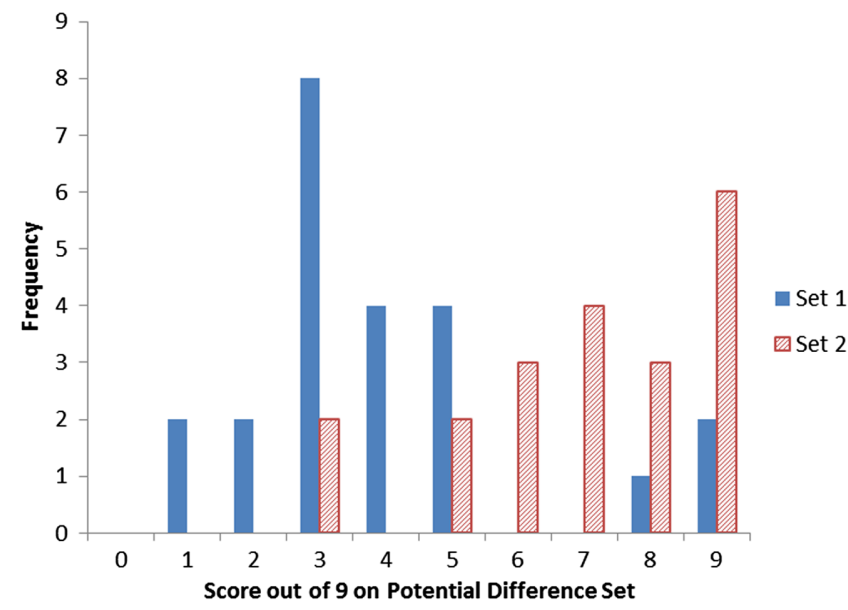

FIG. 5. Distribution of scores, out of 9, potential difference sets 1 and 2. Our criterion for mastering the material was $85 \%$, which meant that a student needed a score of 8 or 9 to master the material. Three out of the 23 students achieved that goal on the first set and moved on to the next topic after being given a chance to view the animated solutions, and therefore are not included in the scores for the second set.

students achieved the mastery goal for potential difference on their first try. When we compare the total score on sets 1 and 2 for superposition for the students who had to do a second set, we find a significant improvement from $37 \%$ to $78 \%$. Once again this improvement can be seen on a question by question basis as well, shown in Fig. 6 . Improvement from the first to second set was found on all nine questions. Seven of them were large gains, and the other two were less substantial due to a ceiling effect. Nine of the 20 remaining students mastered the material by our definition, leaving 11 students to continue on to a third set. Seven mastered the material on their third set, another on the fourth, and 3 students did not finish the experiment due to time constraints.

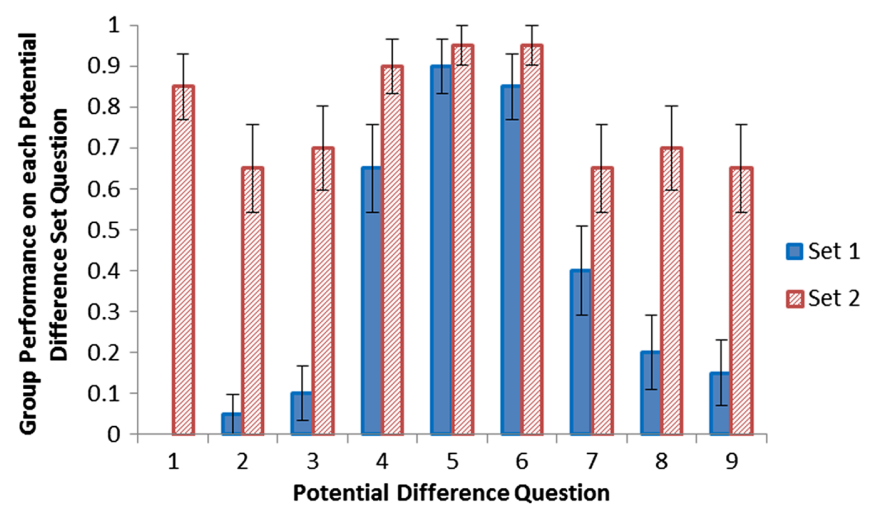

FIG. 6. Scores on questions for sets 1 and 2 of the topic potential difference. This only includes the 20 students who did not master set 1 . None of the students in this group correctly answered question 1 on their first set.
The mastery group students improved from their first try to their second try on both topics. They spent the time in between those tries looking at solution videos. Because of technological difficulties, we were unable to record which animated solutions students watched, but by using recorded time stamps of student submissions and requests for grading, we were able to make a rough estimate of the total amount of time students spent watching solutions in between tries. Figure 7 shows the amount of time spent watching animated solutions after completing the first set of questions on the superposition set for both the mastery and single try groups.

Some student behavior can be noted. The students who got all 8 questions correct watched little to no solutions. Those who missed one question watched between 0 and 3 min of animated solutions, which is notable because most of the solutions were 3-4 min in length. This may indicate students only watched the solution to the single question they got incorrect. In general, time spent watching animated solutions increased with the number of questions missed. The amount of time that the single try group watched solutions surprised us, as they did not have a mastery requirement in order to move on to the next activity. This may speak to the attractiveness of these solutions to students. Single try students did not need to learn from the solutions to move on, but still chose to spend time on them. Figure 8 shows the time spent watching solutions for the mastery group on the potential difference set. Note that no time estimation is available for those who never attempted another set after this, which includes the entire single try group, and those in the mastery group who mastered the potential difference set on their first try. Once again, the time spent rose with the number of questions answered incorrectly. Students spent time watching the solutions, and as was shown previously, improved on their subsequent tries on the question sets.

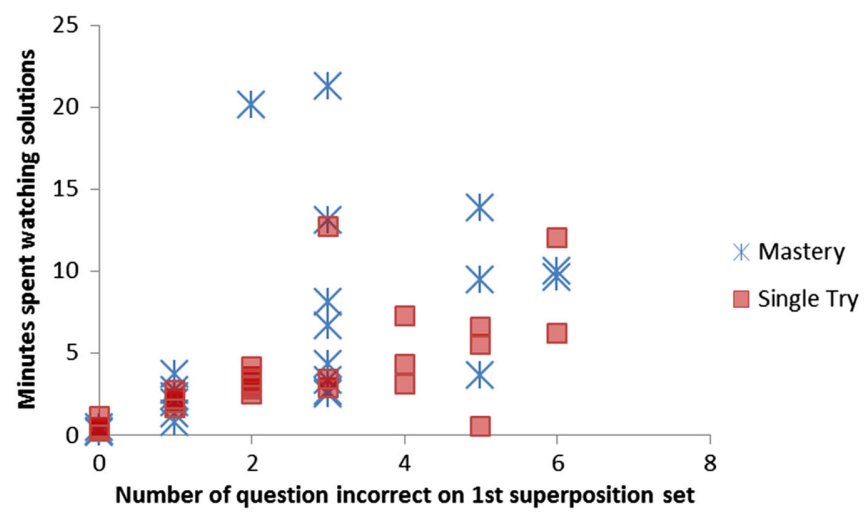

FIG. 7. Scatter plot of time spent watching animated solutions for the first attempt at the superposition set vs the number of questions incorrect on that set. For mastery $r=0.25$, and for single try $r=0.64$. 


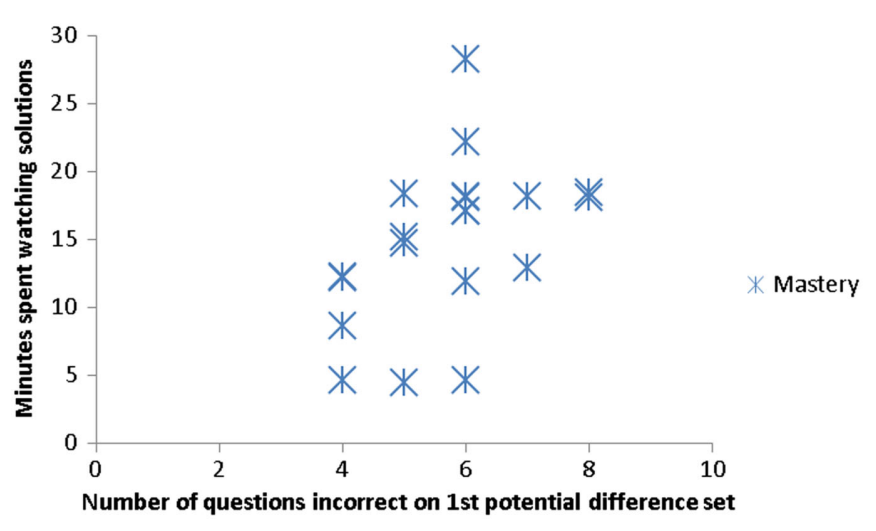

FIG. 8. Scatter plot of time spent watching animated solutions for the first attempt at the potential difference set vs the number of questions incorrect on that set. Shown only are those who were in the mastery group that did not master on their first try, as this estimate was made using the first action on the subsequent set.

Both the mastery group and the single try group spent time watching solution videos during the learning phase of the experiment, and the mastery group exhibited large gains after watching those solutions. Both groups, along with a control group that saw none of the learning materials, attempted a post-test. The mastery treatment group consisted of thirty three people. Of those 33, twenty-four attended the experiment. Of those 24,3 did not finish the mastery materials and therefore did not get the complete treatment, 1 finished the treatment but declined to participate in the post-test, and another 3 decided to leave the post-test before they finished due to other commitments. These 7 will not be included in the post-test analysis. Only the 17 students that completed both the learning materials and did not run out of time on the post-test will be included. Thirty-three students were assigned to the single try group, and 23 attended. Of those 23, all but one finished the posttest in the allotted time. That one student ran out of time and left the experiment early, and will not be included in the post-test analysis. Twenty students were assigned to the control group, and 12 attended the experiment. All 12 completed the post-test in the allotted time. The post-test results are shown in Table II.

TABLE II. Results on post-test with statistical comparisons. Mastery and single try groups significantly outperformed the control group, but did not perform significantly different from each other.

\begin{tabular}{|c|c|c|c|c|c|}
\hline & \multirow[b]{2}{*}{ Post-test Score } & \multicolumn{3}{|c|}{ Compare to control } & $\begin{array}{c}\text { Compare to } \\
\text { single try }\end{array}$ \\
\hline & & tStat & $\mathrm{df}$ & $\mathrm{p}$ & tStat df $\mathrm{P}$ \\
\hline Mastery & $18.4 \pm 1.4$ & 7.45 & 27 & $<0.0001$ & $0.7237>0.2$ \\
\hline Single try & $16.9 \pm 1.4$ & 6.15 & 32 & $<0.0001$ & \\
\hline Control & $4.1 \pm 1.1$ & & & & \\
\hline
\end{tabular}

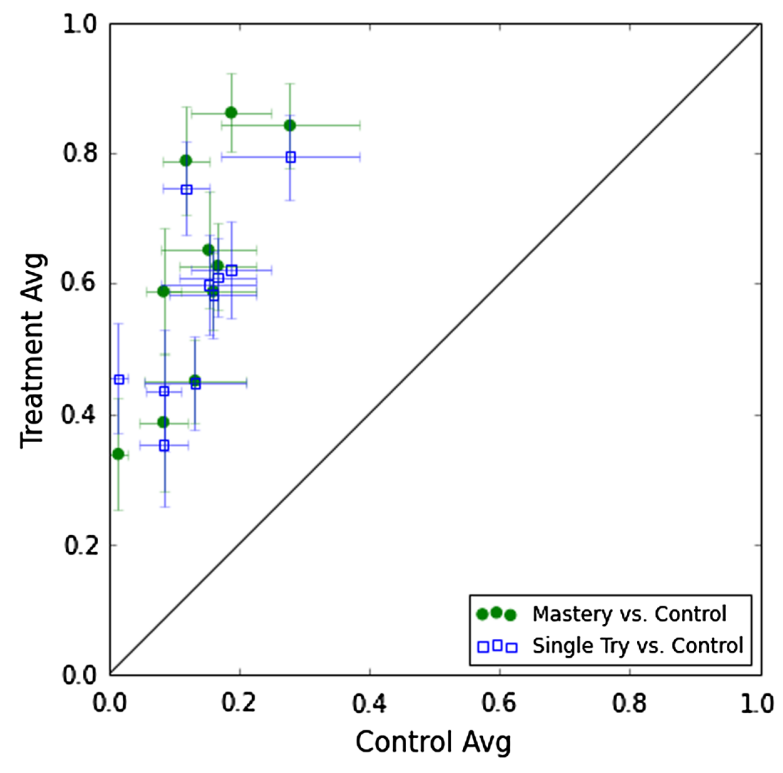

FIG. 9. Normalized student performance on each question in the post-test. All questions lie significantly above the $y=x$ line, where they would be if there was no effect.

The post-test was difficult for the control group, with an average of only 4 of the available 30 points, but serves as a proxy pretest for our treatment groups. The mastery and single try groups were not significantly different, but both performed significantly higher than the control group. That these groups dramatically outperformed the control group suggests that students learned from our materials and were able to apply that knowledge to a post-test where only one question was very near transfer. On a question by question basis, students in the mastery and single try groups outperformed the control group, as shown in Fig. 9. The largest difference between the mastery and single try groups on a single question was on question 8 of the post-test, which asked for a potential difference calculation in a spherical geometry. The mastery group $(0.86 \pm 0.06)$ outperformed the single try group $(0.62 \pm 0.08, \mathrm{P}<0.05)$. This question was very similar to questions seen in the treatment materials. The nearness of transfer from the materials to the post-test may have made this problem easier for the mastery group versus the single try group, especially for those mastery students who had to perform potential difference calculations multiple times over multiple tries.

\section{DISCUSSION}

Students in the mastery and single try groups significantly outperformed a control group on the post-test on planar and spherical potential differences. The post-test only had one question, question 8 , that was very near to what they had seen in the learning materials. The first 7 questions all pertained to potential differences in planar geometries, something they had not been trained on in our experiment, and the students still outperformed the control 
group significantly. It is encouraging to see that both treatment groups were able to apply the knowledge they learned in one context (spherical geometry) to the new context (planar geometry). We saw no significant difference between the mastery and single try groups. We anticipated a difference between the groups because the single try group was not required to perform at a certain level in order to move on, which would lead to them perhaps not watching the solutions as intently or not watching as many of them.

We were surprised by the diligence and effort shown by the single try group during the experiment, especially during the large amounts of time they spent watching the solutions. We expected students to move as quickly as they could to get to the promised office hours. Single try students may have found our solutions very helpful, and given prior studies that show students prefer worked examples to practice problems $[8,9]$, they may have been capitalizing on a chance to see these worked examples that they had not had access to before the experiment. The students made a choice to spend time on these activities, which lowered the amount of time they could spend in the office hours with a tutor. On the other hand, the high level of participation by both groups in watching the solutions may be due to the fact that this was a clinical study, and students set aside time to attend the experiment with the goal of learning a particular subject. This behavior may not hold true if students were to complete the materials on their own time. A 2010 study by Fakcharoenphol, Potter, and Stelzer done at the same institution used worked examples in a nonmastery, nonclinical setting [25]. Less than $45 \%$ of students attempted at least half of the provided materials, and less than $15 \%$ attempted all materials. A $6 \%$ gain was seen, which is much lower than the gain we saw for either treatment group versus the control group. In this experiment we did not ask students why they did or did not watch solutions, but a future experiment could be done that directly measures how often students will use solutions in a clinical setting and investigates student motivations for doing so.

On the post-test, we may not have seen a difference between the single try group and the mastery group because the treatments were too similar in that almost everyone in the single try group watched the solution videos. We are currently implementing mastery style learning on a coursewide scale, replacing standard homework, to get information on how students will interact with this learning style over a longer time period, and to see if the learning is persistent to a normal exam in a course. This should allow us to observe student behavior in a nonclinical setting, as well as test mastery learning in many different topics. We will also compare mastery style learning with the traditional online homework style of unlimited responses in a future clinical experiment.

As regards the mastery group, we have successfully combined formative assessment, learning from multimedia worked examples, and mastery learning via the implementation of narrated animated solution videos in a mastery setting. Students showed significant improvement on their first to second tries on similar sets of problems, attributable to the feedback they got on their performance on the first set coupled with the narrated animated solution videos they viewed between those sets. These improvements were shown in two topics, superposition and electric potential. The mastery setting provided multiple instances of formative assessment and tuned the learning experience to the learner, allowing better prepared students to move on quickly and avoid frustration, and providing more instruction to students who needed more help, resulting in a quick improvement in skills.

While we did not collect data on student affect in this experiment, we remain interested in how students felt and interacted with the project materials. Student emotions have strong effects on their performance in cognitive tasks [26]. Hopelessness and boredom have been shown to have negative correlations with achievement and may lead to reduced attention to materials [27]. One of our main concerns in this project was avoiding student frustration. Several aspects of our implementation should be considered to see if improvements could be made to reduce frustration, as well as to improve learning gain. One is the difficulty of the materials used. The question sets we chose to use were difficult enough to ensure the students had room to grow. This ensured we would be sensitive to an increase. Yet, we remain cautious to include materials that are very difficult for students to grasp, because students may not be able to learn more complex ideas quickly enough to avoid the frustration of having to do many sets before mastery. Initial failure due to too large of a learning requirement may drive students to quit.

The transfer between the sets of materials within a given topic was small, but the questions were not identical. Larger transfer would likely lessen the gains seen after one viewing, but may be fertile grounds for helping students see larger patterns in problem solving. The time spent watching animated solutions rose as the number of missed questions increased, indicating students watched solutions to questions they missed. We suspect students watched the animated solutions not just because they were presented, but because they believed the solutions would help them on the subsequent sets. If transfer is too far, we suspect students may lose that belief, get frustrated, and resort to guessing and cheating to finish a set. We did not see this behavior in our experiment. We did, however, have several students complain of fatigue, and some did not finish the experiment in the time allotted. Unobserved students at home may give up on a set of questions earlier than our volunteers. The difference between question sets in a given topic should not be too large so as to frustrate students. How this difference affects learning, transfer, and frustration should be investigated by challenging students with 
larger changes between sets. The nearness of a subsequent problem must be balanced carefully to keep student trust in the value of watching solutions earnestly.

To further avoid fatigue in students, set length and pass threshold should also be investigated. We used sets of 8 or 9 questions in this experiment, and required students miss no more than one. We tried to account for students making careless mistakes by allowing them to miss one question. This allowance may not have sent the right message to the students, because they had not perfected the material, only performed at a high level. We gave our questions via multiple choice, and included wrong answers that resulted from physics errors or from equation hunting, not from careless math errors. This should cut down on false negatives of students not moving on to the next topic for the wrong reason. The long set length required students to reproduce long calculations that they may have gotten correct on previous sets between chances at assessment. This fatigued many students, and created more opportunities for students to make careless mistakes, which prevented them from mastering the material. In the future, shorter sets with a $100 \%$ pass threshold may allow students to focus on fewer skills at a time, as well providing students with more instances to receive the positive feedback of mastering material, while reducing the odds that a student will not move on the for the wrong reason, such as a math error, and sending the correct message to students that they have to master a topic before moving on. In the research to come we will investigate different amounts of transfers between sets, as well as different lengths of the material.

\section{ACKNOWLEDGEMENTS}

We would like to thank the reviewers of this paper, whose insightful comments have greatly improved the quality of this paper. This work was partially funded by National Science Foundation DUE Grant No. 0817185.
[1] For reviews see, R. K. Atkinson, S. J. Derry, A. Renkl, and D. Wortham, Learning from examples: Instructional principles from the worked examples research, Rev. Educ. Res. 70, 181 (2000); J. Sweller, J. van Merriënboer, and F. Paas, Cognitive architecture and instructional design, Educ. Psychol. Rev. 10, 251 (1998).

[2] J. Sweller, Cognitive load during problem solving: Effects on learning, Cogn. Sci. 12, 257 (1988).

[3] J. E. Tuovinen and J. Sweller, A comparison of cognitive load associated with discovery learning and worked examples, J. Educ. Psychol. 91, 334 (1999).

[4] J. Sweller and P. Chandler Evidence for cognitive load theory, Cognit. Instr. 8, 351 (1991).

[5] X. Zhu and H.A. Simon, Learning mathematics from examples and by doing, Cognit. Instr. 4, 137 (1987).

[6] M. W. Carroll, Using worked examples as an instructional support in the algebra classroom, J. Educ. Psychol. 86, 360 (1994).

[7] M. T. H. Chi, M. Bassok, M. W. Lewis, P. Reimann, and R. Glaser, Self-explanations: How students study and use examples in learning to solve problems, Cogn. Sci. 13, 145 (1989).

[8] P. Pirolli and J. R. Anderson, The role of learning from examples in the acquisition of recursive programming skills, Canad. J. Psychol. 39, 240 (1985).

[9] J. R. Anderson, R. Farrell, and R. Sauers, Learning to program in LISP, Cogn. Sci. 8, 87 (1984).

[10] S. Kalyuga, P. Ayres, P. Chandler, and J. Sweller, The expertise reversal effect, Educ. Psychol. 38, 23 (2003).

[11] W. G. Chase and H. A. Simon, Perception in chess, Cogn. Psychol. 4, 55 (1973).
[12] M. Ward and J. Sweller, Structuring effective worked examples, Cognit. Instr. 7, 1 (1990).

[13] R. C. Clark, F. Nguyen, and J. Sweller, Efficiency in Learning: Evidence-Based Guidelines to Manage Cognitive Load (John Wiley \& Sons, New York, 2011).

[14] R. E. Mayer, Multimedia Learning (Cambridge University Press, Cambridge, England, 2001).

[15] W. Schnotz, Towards an integrated view of learning from text and visual displays, Educ. Psychol. Rev. 14, 101 (2002).

[16] R. E. Mayer and R. Moreno, Nine ways to reduce cognitive load in multimedia learning, Educ. Psychol. 38, 43 (2003).

[17] T. Stelzer, G. Gladding, J. Mestre, and D. T. Brooks, Comparing the efficacy of multimedia modules with traditional textbooks for learning introductory physics content, Am. J. Phys. 77, 184 (2009).

[18] Z. Chen, T. Stelzer, and G. Gladding, Using multimedia modules to better prepare students for introductory physics lecture, Phys. Rev. ST Phys. Educ. Res. 6, 010108 (2010).

[19] P. Black and D. Wiliam, Assessment and classroom learning, Assess. Educ. Princ. Pol. Pract. 5, 7 (1998).

[20] H. L. Roediger and J. D. Karpicke, The power of testing memory: Basic research and implications for educational practice, Perspect. Psychol. Sci. 1, 181 (2006).

[21] A.C. Butler and H.L. Roediger, Feedback enhances the positive effects and reduces the negative effects of multiple-choice testing, Mem. Cogn. 36, 604 (2008).

[22] C. L. C. Kulik, J. A. Kulik, and R. L. Bangert-Drowns, Effectiveness of mastery learning programs: A metaanalysis, Rev. Educ. Res. 60, 265 (1990).

[23] See Supplemental Material at http://link.aps.org/ supplemental/10.1103/PhysRevSTPER.11.010103 for the question sets used in the learning phase, the final frame of 
some representative narrated animated solutions, and the post-test.

[24] Z. Chen and G. Gladding, How to make a good animation: A grounded cognition model of how visual representation design affects the construction of abstract physics knowledge, Phys. Rev. ST Phys. Educ. Res. 10, 010111 (2014).

[25] W. Fakcharoenphol, E. Potter, and T. Stelzer, What students learn when studying physics practice exam problems, Phys. Rev. ST Phys. Educ. Res. 7, 010107 (2011).
[26] A. Gupta, Integrating emotions into fine-grained accounts of students' reasoning presented at the Physics Education Research Conference 2013, Portland, OR, 2013, http:// www.compadre.org/Repository/document/ServeFile.cfm? $\mathrm{ID}=13076 \& \mathrm{DocID}=3621$.

[27] R. Pekrun, T. Goetz, W. Titz, and R. P. Perry, Academic emotions in students' self regulated learning and achievement: A program of qualitative and quantitative research, Educ. Psychol. 37, 91 (2002). 